\title{
Adenine base editing to mimic or correct disease mutations in rodents
}

\author{
Ruotong Ren ${ }^{1,2,3}$, Juan Carlos Izpisua Belmonte ${ }^{4 \bowtie}$, Guang-Hui Liu ${ }^{1,2,3,5 \bowtie}$ \\ ${ }^{1}$ Advanced Innovation Center for Human Brain Protection, National Clinical Research Center for Geriatric Disorders, Xuanwu \\ Hospital of Capital Medical University, Beijing 100053, China \\ 2 National Laboratory of Biomacromolecules, CAS Center for Excellence in Biomacromolecules, Institute of Biophysics, \\ Chinese Academy of Sciences, Beijing 100101, China \\ ${ }^{3}$ University of Chinese Academy of Sciences, Beijing 100049, China \\ ${ }^{4}$ Gene Expression Laboratory, Salk Institute for Biological Studies, 10010 North Torrey Pines Road, La Jolla, California 92037, \\ USA \\ ${ }^{5}$ Institute of Stem cell and Regeneration, Chinese Academy of Sciences, Beijing 100101, China \\ $\bowtie$ Correspondence: belmonte@salk.edu (J. C. I. Belmonte), ghliu@ibp.ac.cn (G.-H. Liu)
}

Cytidine base editors (CBEs, rAPOBEC1-nCas9-UGI) and adenine base editors (ABEs, TadA-Tad $A^{*}$-nCas9) are newly developed genome-editing tools that have enabled highly efficient base conversions $(C \cdot G$ to $T \cdot A$ or $A \cdot T$ to $G \cdot C$ ) at designated target sites (Komor et al., 2016; Gaudelli et al., 2017). Both types of base editors enzymatically catalyze deamination of target bases and respectively replace $C$ with $\mathrm{U}$ or $\mathrm{A}$ with I.

After CBEs were first reported, Kim et al. and Liang et al. quickly followed with studies that demonstrated independently the potential of using CBEs to generate point mutation mouse models (Liang et al., 2017a, b; Kim et al., 2017). Liang et al. also showed the effectiveness of CBEs in correcting $\beta$-thalassemia point mutations in human embryos (Liang et al., 2017a, b). The advent of ABE has stimulated similarly spirited research efforts. Four groups have now independently shown that $A B E s$ can generate $A-$ to-G point mutation disease mouse and rat models (Ryu et al., 2018; Liu et al., 2018). In addition, Ryu et al. used a viral vector to deliver the $A B E$ into muscles of adult mice so as to correct mutations in the Dunchenne muscular dystrophy (DMD) gene (Ryu et al., 2018). DMD is a progressive and degenerative neuromuscular disease with no effective treatment. The mutation corrected in the study by Ryu et al. was generated by a CBE and not found in DMD patients (Kim et al. 2017). In this issue of Protein and Cell, Liang et al. used ABEs to directly link Dunchenne muscular dystrophy with $D M D$ gene splice site mutations that have been found in DMD patients (Liang et al., 2018). The three splice site mutations they identified from the DMD mutation database have no cellular or animal models. The group therefore developed the AI-MAST (ABE-induced mRNA splicing defect) method to target specific splice sites of $D M D$.
Following delivery into mouse zygotes, the $A B E$ was able to edit the targeted adenine base with high efficiency as was observed in both mouse blastocysts and F0 mice. Moreover, DMD splice site mutant mice displayed obvious muscle weakness and marked upregulation of creatine kinase (CK), which are characteristic symptoms of DMD and indicate a successful one-step generation of DMD disease models using AI-MAST. In addition, the list of potential disease splice site mutations that are targetable by AI-MAST should also prove valuable to researchers studying disease pathogenesis and searching for new therapeutics. Additionally, Yang et al. reported the efficient generation of a hereditary tyrosinemia type I disease model in mice and a glycogen storage disease type II (GSD II; Pompe disease) model in rats using ABE technology in the same issue of Protein and Cell (Yang et al., 2018). It is the first report to use base editors to generate disease model in rats. Moreover, they showed that using chemically modified gRNA could improve the editing efficiency of ABE by increasing gRNA stability. Through fusion of adenosine deaminase to SaCas9 and SpCas9 variants, Yang et al. further expanded the targeting scope of ABEs in both cell lines and animals.

Both CBEs and ABEs can in theory precisely generate and/or repair point mutations without inducing DNA double strand breaks (DSBs). However, removal of base $U$ by the uracil DNA glycosylase (UDG) in eukaryotic cells would result in uncontrolled C-to-A/G conversions and DNA DSBs (Liang et al., 2017a, b; Kim et al., 2017; Liu et al., 2018). In contrast, the lack of enzymatic removal of base I would mean potentially more efficient and specific A-to-G conversions by ABEs without detectable DNA DSBs (Ryu et al., 2018; Liu et al., 2018). While the power of ABEs in generating point mutation animal models and correcting disease 
mutations is clearly evident from the four studies, their specificity remains to be determined, especially in studies using unbiased genome-wide off-target detection platforms. Detection of base editor off-targets differs from that for CRISPR/Cas9 and presents its own challenges. A new methodology that can accurately and efficiently detect genome-wide off-targets of ABEs is therefore sorely needed. Only with such technologies can ABE specificity be properly and effectively improved.

\section{ACKNOWLEDGEMENTS}

This work was supported by the Strategic Priority Research Program of the Chinese Academy of Sciences (XDA16010100), the National Key Research and Development Program of China (2015CB964800), and UCAM, Spain.

\section{OPEN ACCESS}

This article is distributed under the terms of the Creative Commons Attribution 4.0 International License (http://creativecommons.org/ licenses/by/4.0/), which permits unrestricted use, distribution, and reproduction in any medium, provided you give appropriate credit to the original author(s) and the source, provide a link to the Creative Commons license, and indicate if changes were made.

\section{REFERENCES}

Gaudelli NM, Komor AC, Rees HA, Packer MS, Badran AH, Bryson DI, Liu DR (2017) Programmable base editing of $A \cdot T$ to $G \cdot C$ in genomic DNA without DNA cleavage. Nature 551:464-471
Kim K, Ryu SM, Kim ST, Baek G, Kim D, Lim K, Chung E, Kim S, Kim JS (2017) Highly efficient RNA-guided base editing in mouse embryos. Nat Biotechnol 35:435-437

Komor AC, Kim YB, Packer MS, Zuris JA, Liu DR (2016) Programmable editing of a target base in genomic DNA without double-stranded DNA cleavage. Nature 533:420-424

Liang P, Sun H, Sun Y, Zhang X, Xie X, Zhang J, Zhang Z, Chen Y, Ding $C$, Xiong $Y$ et al (2017a) Effective gene editing by highfidelity base editor 2 in mouse zygotes. Protein Cell 8:601-611

Liang P, Ding C, Sun H, Xie X, Xu Y, Zhang X, Sun Y, Xiong Y, Ma W, Liu $Y$ et al (2017b) Correction of $\beta$-thalassemia mutant by base editor in human embryos. Protein Cell 8:811-822

Liang P, Sun H, Zhang X, Xie X, Zhang J, Bai Y, Ouyang X, Zhi S, Xiong Y, Ma W et al (2018) Effective and precise adenine base editing in mouse zygotes. Protein Cell. https://doi.org/10.1007/ s13238-018-0566-Z

Liu Z, Lu Z, Yang G, Huang S, Li G, Feng S, Liu Y, Li J, Yu W, Zhang $Y$ et al (2018) Efficient generation of mouse models of human diseases via ABE- and BE-mediated base editing. Nat Commun 9:2338

Ryu SM, Koo T, Kim K, Lim K, Baek G, Kim ST, Kim HS, Kim DE, Lee $\mathrm{H}$, Chung $\mathrm{E}$ et al (2018) Adenine base editing in mouse embryos and an adult mouse model of Duchenne muscular dystrophy. Nat Biotechnol 36:536

Yang L, Zhang X, Wang L, Yin S, Zhu B, Xie L, Duan Q, Hu H, Zheng $R$, Wei $Y$ et al (2018) Increasing targeting scope of adenosine base editors in mouse and rat embryos through fusion of TadA deaminase with Cas9 variants. Protein Cell. https://doi.org/10. 1007/s13238-018-0568-x 